\title{
Editorial for Special Issue: COCOA2014
}

\author{
Zhao Zhang ${ }^{1}$
}

Eight papers published in this special issue are selected from 49 presentations in the 8th Annual International Conference on Combinatorial Optimization and Applications. They cover the algorithm and complexity aspect of various topics in classic and new combinatorial optimization problems, including directed Steiner tree, group testing, popular matching, online leasing and online matching, etc. These papers are required to be substantially different from their conference versions. They have passed a regular review process for this journal. We would like to express our gratitude to all reviewers for their hard work and the authors for their contribution.

$凶$ Zhao Zhang hxhzz@163.com

1 College of Mathematics Physics and Information Engineering, Zhejiang Normal University, Jinhua 321004, Zhejiang, China 